\title{
Thermo-tolerant phosphate-solubilizing microbes for multi-functional biofertilizer preparation
}

\author{
Cheng-Hsiung Chang ${ }^{a}$, Shang-Shyng Yang ${ }^{a, b, *}$ \\ a Institute of Microbiology and Biochemistry, National Taiwan University, Taipei 10617, Taiwan \\ ${ }^{\mathrm{b}}$ Department of Biochemical Science and Technology, National Taiwan University, Taipei 10617, Taiwan
}

\section{A R T I C L E I N F O}

\section{Article history:}

Received 4 May 2008

Received in revised form 29 August 2008

Accepted 2 September 2008

Available online 31 October 2008

\section{Keywords:}

Thermo-tolerant phosphate-solubilizing microbes

Soluble phosphorus content

Biofertilizer

Maturity

\begin{abstract}
A B S T R A C T
In order to prepare the multi-functional biofertilizer, thermo-tolerant phosphate-solubilizing microbes including bacteria, actinomycetes, and fungi were isolated from different compost plants and biofertilizers. Except Streptomyces thermophilus J57 which lacked pectinase, all isolates possessed amylase, CMCase, chitinase, pectinase, protease, lipase, and nitrogenase activities. All isolates could solubilize calcium phosphate and Israel rock phosphate; various isolates could solubilize aluminum phosphate, iron phosphate, and hydroxyapatite. During composting, biofertilizers inoculated with the tested microbes had a significantly higher temperature, ash content, $\mathrm{pH}$, total nitrogen, soluble phosphorus content, and germination rate than non-inoculated biofertilizer; total organic carbon and carbon-to-nitrogen ratio showed the opposite pattern. Adding these microbes can shorten the period of maturity, improve the quality, increase the soluble phosphorus content, and enhance the populations of phosphate-solubilizing and proteolytic microbes in biofertilizers. Therefore, inoculating thermo-tolerant phosphate-solubilizing microbes into agricultural and animal wastes represents a practical strategy for preparing multi-functional biofertilizer.
\end{abstract}

(c) 2008 Elsevier Ltd. All rights reserved.

\section{Introduction}

In addition to nitrogen, phosphorus is one of the most important nutrients for plant growth. Phosphorus contributes to the biomass construction of micronutrients, the metabolic process of energy transfer, signal transduction, macromolecular biosynthesis, photosynthesis, and respiration chain reactions (Shenoy and Kalagudi, 2005). Unfortunately, phosphorus is one of the least available and the least mobile mineral nutrients for plants in the soil (Takahashi and Anwar, 2007). Many soils have a high reserve of total phosphorus accounting for about $0.05 \%$ of soil content on average; however, only $0.1 \%$ of the total phosphorus is available to plants (Zou et al., 1992). Therefore, phosphatic fertilizers, such as costly chemical fertilizers that contain large amounts of soluble phosphorus, have been applied to the agricultural fields to maximize production (Del Campillo et al., 1999; Shenoy and Kalagudi, 2005). However, the soluble phosphorus in phosphatic fertilizers is easily and rapidly precipitated to insoluble forms with cations such as $\mathrm{Ca}^{2+}, \mathrm{Fe}^{3+}, \mathrm{Al}^{3+}, \mathrm{Co}^{2+}$, or $\mathrm{Zn}^{2+}$, or adsorbed to calcium carbonate, aluminum oxide, iron oxide, and aluminum silicate,

\footnotetext{
* Corresponding author. Address: Department of Biochemical Science and Technology, National Taiwan University, Taipei 10617, Taiwan. Tel.: +886 2 33664456; fax: +886223679827.

E-mail address: ssyang@ntu.edu.tw (S.-S. Yang).
}

depending on the particular properties of the soil (Del Campillo et al., 1999; Wakelin et al., 2004). This transformation decreases the efficiency with which soluble phosphorus can be taken up by the plants and decreases the effectiveness of the fertilizer, resulting in the application of increasing amounts of phosphatic fertilizers to agricultural fields. The unmanaged use of phosphatic fertilizers has increased agricultural costs and instigated a variety of environmental problems (Del Campillo et al., 1999). Therefore, the concept of adding phosphate-solubilizing microbes to fertilizers as providers of soluble phosphorus presents an economically and environmentally promising strategy.

Phosphate-solubilizing microbes play fundamental roles in biogeochemical phosphorus cycling in natural and agricultural ecosystems. Phosphate-solubilizing microbes can transform the insoluble phosphorus to soluble forms $\mathrm{HPO}_{4}^{2-}$ and $\mathrm{H}_{2} \mathrm{PO}_{4}^{-}$by acidification, chelation, exchange reactions, and polymeric substances formation (Delvasto et al., 2006). Therefore, the use of phosphate-solubilizing microbes in agricultural practice would not only offset the high cost of manufacturing phosphatic fertilizers but would also mobilize insoluble phosphorus in the fertilizers and soils to which they are applied (Rodríguez and Fraga, 1999). Application of the phosphate-solubilizing microbes Agrobacterium, Bacillus, Enterobacter, Pseudomonas, Aspergillus, Trichoderma and Glomus around the roots of plants, in soils, and in fertilizers has been shown to release soluble phosphorus, promote plant growth, and protect plants from pathogen infection (Rodríguez and Fraga, 
1999; Rudresh et al., 2005; Zayed and Abdel-Motaal, 2005a,b; Biswas and Narayanasamy, 2006; Ouahmane et al., 2007).

Compost preparation is very popular in Taiwan and Asian countries as a means of recycling agricultural by-products, poultry, livestock, industrial, and municipal wastes. The application of compost to soil can improve the soil quality, supply nutrients to plants and soil microbes, and reduce environmental pollution (Yang, 2003), and inoculation of the appropriate microbes during compost preparation can shorten the maturity period and improve the quality of compost (Yang and Chen, 2003; Zayed and Abdel-Motaal, 2005a,b; Biswas and Narayanasamy, 2006). However, all phosphate-solubilizing microbes studied and applied to date have been mesophiles that could only be used under mesophilic conditions. These types of microbes are not appropriate for biofertilizer preparation at the high temperatures (over $50^{\circ} \mathrm{C}$ ) that occur during the first stage of composting (Yang and Chen, 2003).

In Taiwan, more than $60 \%$ of farmland soils are acidic and their organic contents are less than $2 \%$ due to the high temperature, frequent rainfall, and intensive agriculture (Huang, 1991). Therefore, about 20 tons of organic matter per hectare per year is used to reduce the mineralization and phosphorus immobilization in soil, to improve the soil quality, and to recover the soil fertility (Lee, 1999). In 2006, the annual animal solid waste production was 13.1 million tons. Chicken waste was the major component, accounting for $44.3 \%$, followed by hog waste, which accounted for $39.4 \%$ (Yang et al., 2003; Council of Agriculture/Taiwan, 2007). These animal wastes can be mixed with various biological bulking materials, such as agricultural by-products, crop residue, tea residue, sawdust, vegetable-market waste, waste mushroom media, food processing waste, and municipal refuse, and then inoculated with the appropriate microbes to prepare biofertilizers with high soluble phosphorus content (Yang, 2003).

Composting is a microbiological process that transforms various organic wastes into biofertilizers and soil conditioners. Microbes that survive compost preparation can adapt to wide temperature variations, tolerate high temperatures, and have various enzyme activities responsible for decomposing complex organic wastes (Yang, 2003). Therefore, we isolated thermotolerant multi-functional phosphate-solubilizing microbes from different stages of compost preparation and from different biofertilizers, and investigated the effects of these microbes on the maturity, quality, and phosphorus-movement of compost during biofertilizer preparation.

\section{Methods}

\subsection{Raw materials}

The agricultural and animal wastes were kindly provided from one compost plant located in Taoyuan County, Taiwan. The wastes were crushed into the granules roughly below $40.0 \mathrm{~mm}$ in diameter and contained $15 \%$ chicken waste, $15 \%$ Chinese herbal residue, $35 \%$ sawdust, $10 \%$ tea residue, $10 \%$ paper pulp, and $15 \%$ mixture of food proceeding sludge and waste form poultry and livestock slaughter houses. The raw materials had $\mathrm{pH} 6.9 \pm 0.1$, moisture content of $51.9 \pm 1.2 \%$, ash content of $39.3 \pm 6.0 \%$, total organic carbon of $47.0 \pm 1.8 \%$, total nitrogen of $1.83 \pm 0.08 \%$, carbon-to-nitrogen $(\mathrm{C} / \mathrm{N})$ ratio of $25.8 \pm 1.6$, soluble phosphorus content of $4.1 \pm 0.3 \mathrm{~g} \mathrm{~kg}^{-1}$, total phosphorus content of $33.3 \pm 0.3 \mathrm{~g} \mathrm{~kg}^{-1}$, and alfalfa seed germination rate of $54.7 \pm 2.3 \%$.

\subsection{Tested microbes}

Thermo-tolerant phosphate-solubilizing microbes including three bacteria (Bacillus coagulans C45, Bacillus licheniformis A3,
Bacillus smithii F18), one actinomycete (Streptomyces thermophilus $\mathrm{J} 57$ ), and one fungus (Aspergillus fumigatus 04) were isolated from different compost plants and biofertilizers. Brevibacillus borstelensis SH168, Streptomyces thermonitrificans NTU-88, and A. fumigatus NTU-132 were used as reference strains (Chang et al., 2001; Yang and Chen, 2003; Tsai et al., 2007). The bacteria and actinomycetes were cultivated in nutrient agar; while fungi were cultivated in potato dextrose agar.

\subsection{Enzyme activity}

Enzyme activity was measured by plate and broth assay at 25 and $50{ }^{\circ} \mathrm{C}$ for 5 and 10 days, respectively. $\alpha$-Amylase activity was assayed by soluble starch-yeast extract medium (Tsai et al., 2007; Kammoun et al., 2008). CMCase activity was determined by Mandels-Reese medium with carboxymethylcellulose as the sole carbon source (Tsai et al., 2007; Lee et al., 2008). Protease activity was assayed by skim milk medium (Tsai et al., 2007; Mahanta et al., 2008). Chitinase and pectinase activities were determined in Mandels-Reese medium with chitin and pectin as the sole carbon source, respectively (Mandels et al., 1981; Tsai et al., 2007). Lipase activity was measured with tributyrin medium (Tsai et al., 2007). Nitrogenase activity was determined in Winogradsky's nitrogen-free mineral medium. Excluding nitrogenase, the halo ratios of clear zone (CZ)/ colony size (CS) were calculated as enzyme activity indices (EAI), while CS was used to evaluate nitrogenase activity (Aquilanti et al., 2004).

\subsection{Inorganic phosphate-solubilizing activity}

Inorganic phosphate-solubilizing activity was measured by plate and broth assay at 25 and $50^{\circ} \mathrm{C}$, respectively. Pikovskaya's medium (PVK) was used to measure calcium phosphate $\left[\mathrm{Ca}_{3}\left(\mathrm{PO}_{4}\right)_{2}\right]$-solubilizing activity, while PVK replaced with $5 \mathrm{~g}$ of aluminum phosphate $\left(\mathrm{AlPO}_{4}\right)$, iron phosphate $\left(\mathrm{FePO}_{4}\right)$, hydroxyapatite (HA), or Israel rock phosphate (RP) from tricalcium phosphate as the sole phosphorus source was used to measure $\mathrm{AlPO}_{4}, \mathrm{FePO}_{4}$, HA, and RP-solubilizing activities, respectively (Reddy et al., 2002). Plate assays were performed for 5 days, and the phosphate-solubilizing activity index (PSAI = CZ/CS) was calculated (Nautiyal, 1999; Chang et al., 2001). Broth assays were performed for 10 days, and $\mathrm{pH}$ and soluble phosphorus in the supernatant were determined with a $\mathrm{pH}$ meter (Horiba $\mathrm{pH}$ meter F-21) and by the colorimetric molybdate blue method, respectively (Olsen and Sommers, 1982; Nautiyal, 1999).

\subsection{Biofertilizer preparation}

Each gram of dry raw material was inoculated with tested microbes at about $1 \times 10^{5}$ CFU cells, spores, and fragments of bacteria, actinomycetes, and fungi, respectively, at an initial moisture content of $60-65 \%$ in a 35-1 impermeable plastic container (37$\mathrm{cm}$ inner diameter at the top, 28-cm inner diameter at the bottom, and $45-\mathrm{cm}$ height). The compost was turned over every three to four days for 56 days. Non-inoculated raw material was used as the control (Yang and Chen, 2003).

\subsection{Microbial populations}

Microbial populations were determined by serial dilution and pour plate methods. Mesophilic microbes were grown at $25^{\circ} \mathrm{C}$ and thermo-tolerant microbes were grown at $50{ }^{\circ} \mathrm{C}$ for five days. Bacteria, actinomycetes, fungi, phosphate-solubilizing bacteria, phosphate-solubilizing actinomycetes, phosphate-solubilizing fungi, and proteolytic microbes were counted on nutrient agar (NA), glycerol-yeast extract agar, Rose-Bengal agar, PVK, glycerol-yeast 
extract agar replaced with $5 \mathrm{~g}$ of $\mathrm{Ca}_{3}\left(\mathrm{PO}_{4}\right)_{2}$ from $\mathrm{KH}_{2} \mathrm{PO}_{4}$ as the sole phosphorus source, Rose-Bengal agar with $5 \mathrm{~g}$ of $\mathrm{Ca}_{3}\left(\mathrm{PO}_{4}\right)_{2}$ from $\mathrm{KH}_{2} \mathrm{PO}_{4}$ as the sole phosphorus source, and skim milk agar, respectively (Reddy et al., 2002; Zayed and Abdel-Motaal, 2005a,b; Chang and Yang, 2006; Tsai et al., 2007).

\subsection{Analysis}

Temperatures were determined in situ with a mercury thermometer. pH was measured directly in the tested composts or in $1: 10\left(\mathrm{w} \mathrm{v}^{-1}\right)$ compost to water suspensions using a $\mathrm{pH}$ meter. Moisture content was determined by drying samples at $105^{\circ} \mathrm{C}$ to a constant weight. Ash content was measured after heating at $550{ }^{\circ} \mathrm{C}$ for $24 \mathrm{~h}$. Total organic carbon was estimated using a TOC5000 A total organic carbon analyzer (Code HI 8424 C, Shimadzu, Japan; Hegde et al., 2003). Total nitrogen was measured by a modified Kjeldahl method (Yang et al., 1991). Alfalfa seed germination rate was determined as described by Pai et al. (2003). The dry weight loss was the percentages of dry weight decreasing during preparation. The dry weights of composts were counted as fresh weight minus moisture content (Chang and Yang, 2006). Color and odor were judged personally. Total phosphorus was measured by the colorimetric molybdate blue method following Kjeldahl digestion. Soluble phosphorus was extracted using Bray no. 1 extraction solution $\left(0.025 \mathrm{~N} \mathrm{HCl}\right.$ and $\left.0.03 \mathrm{~N} \mathrm{NH}_{4} \mathrm{~F}\right)$ and determined by the colorimetric molybdate blue method (Olsen and Sommers, 1982; Chang and Yang, 2006).

\subsection{Statistical analysis}

All experiments were carried out in triplicate or more. All data are reported as means \pm SD (standard deviation). The IndependentSamples $t$-test was used to compare means and the variance homogeneity determination (ANOVA) was conducted with the General Linear Model using type II sum of squares and Tukey's Honestly Significant Difference $(P=0.05)$ using statistical analysis system software (SAS Institute, 2002).

\section{Results and discussion}

\subsection{Isolation of phosphate-solubilizing microbes}

The composts were gathered from one hog waste compost plant, five chicken waste compost plants, two cow waste compost plants, two commercial biofertilizers, and two biofertilizer preparations consisting of rice straw, hog, and poultry wastes treated with polyelectrolyte oxygen detoxifier. The temperatures of chicken, cow, and hog waste composts, and biofertilizers were 19-84, $31-77,19-74$, and $26-62{ }^{\circ} \mathrm{C}$, respectively. The total phosphorus contents were $23-92,23-29,10-55$, and $12-27 \mathrm{~g} \mathrm{~kg}^{-1}$, respectively. Therefore, all the samples were the suitable sources to isolate thermo-tolerant phosphate-solubilizing microbes, and 977 phosphate-solubilizing microbes were isolated. It included 202 mesophilic bacteria, 621 thermo-tolerant bacteria, 40 mesophilic actinomycetes, 49 thermo-tolerant actinomycetes, 42 mesophilic fungi, and 23 thermo-tolerant fungi. After performing a series tests of phosphate-solubilizing and enzyme activities, three bacteria ( $B$. coagulans $\mathrm{C} 45$, B. licheniformis A3, B. smithii F18), one actinomycete (S. thermophilus J57), and one fungus (A. fumigatus 04 ) isolates were selected. At $50^{\circ} \mathrm{C}$ in PVK medium, $B$. coagulans $C 45$ had yellowish-white and formed round colonies, B. licheniformis A3 formed white and hairlike colonies, B. smithii F18 formed white and round colonies, S. thermophilus J57 had yellowish-brown and round colonies, and A. fumigatus 04 formed yellow and round colonies.

\subsection{Thermo-tolerant test}

Bacillus coagulans $\mathrm{C} 45$, B. licheniformis A3, and B. smithii F18 grew on nutrient agar at $25-75^{\circ} \mathrm{C}$, while $S$. thermophilus J57 grew at $25-65^{\circ} \mathrm{C}$. Aspergillus fumigatus 04 grew on potato dextrose agar at $25-65^{\circ} \mathrm{C}$. Bacillus coagulans $\mathrm{C} 45$, B. smithii F18, S. thermophilus $\mathrm{J} 57$, and $S$. thermonitrificans NTU-88 had the significantly $(P<0.001)$ largest colonies at $50{ }^{\circ} \mathrm{C}$; however, A. fumigatus 04 and $A$. fumigatus NTU-132 had the significantly $(P<0.001)$ largest colonies at $25^{\circ} \mathrm{C}$. Bacillus licheniformis A3 and $\mathrm{Bv}$. borstelensis SH168 formed swarming colonies at $50{ }^{\circ} \mathrm{C}$. Bacillus coagulans C45, $B$. licheniformis $\mathrm{A} 3$, and $B$. smithii $\mathrm{F} 18$ had cell densities of $1.4 \pm 0.2 \times 10^{10}, 1.4 \pm 0.1 \times 10^{10}$, and $1.3 \pm 0.3 \times 10^{10} \mathrm{CFU} \mathrm{m}^{-1}$ at $50{ }^{\circ} \mathrm{C}$ for 2 days incubation in PVK broth, respectively; while the cell densities were $9.3 \pm 0.8 \times 10^{9}, 8.5 \pm 1.8 \times 10^{9}$, and $2.3 \pm 0.2 \times$ $10^{9} \mathrm{CFU} \mathrm{ml} \mathrm{m}^{-1}$ at $25^{\circ} \mathrm{C}$. Streptomyces thermophilus $\mathrm{J} 57$ and $A$. fumigatus $\mathrm{O} 4$ had the mycelium dry weights of $5.3 \pm 0.2$ and $3.1 \pm 0.3 \mathrm{mg}$ $\mathrm{ml}^{-1}$, respectively at $50{ }^{\circ} \mathrm{C}$ in PVK broth where tricalcium phosphate was replaced by RP as the sole phosphorus source. The mycelium dry weights were $4.2 \pm 0.4$ and $6.8 \pm 0.2 \mathrm{mg} \mathrm{ml}^{-1}$ at $25^{\circ} \mathrm{C}$, respectively. The phosphate-solubilizing bacteria had been isolated from the root-free soil, rhizosphere, and rhizoplane in alkaline soils (Johri et al., 1999), the rhizosphere of chickpea and alkaline soils (Nautiyal et al., 2000), and coastal sand dune legumes (Arun and Sridhar, 2005). However, they grew and solubilized phosphate at $35-45^{\circ} \mathrm{C}$ only. In this study, B. coagulans C45, B. licheniformis A3, B. smithii F18, S. thermophilus J57, Bv. borstelensis SH168, and $S$. thermonitrificans NTU-88 were thermo-tolerant microbes and grew faster at $50{ }^{\circ} \mathrm{C}$ than at $25^{\circ} \mathrm{C}$, while $A$. fumigatus 04 and $A$. fumigatus NTU-132 were mesophilic microbes and grew faster at $25^{\circ} \mathrm{C}$ than at $50{ }^{\circ} \mathrm{C}$. All the tested strains could tolerate high temperatures to grow and solubilize phosphate during composting.

\subsection{Enzyme activity}

The enzyme activities of tested isolates are shown in Tables 1 and 2. At $50{ }^{\circ} \mathrm{C}, B$. coagulans $\mathrm{C} 45, \mathrm{~B}$. licheniformis $\mathrm{A} 3$, B. smithii $\mathrm{F} 18$, $B v$. borstelensis SH168, S. thermonitrificans NTU-88, A. fumigatus 04 and A. fumigatus NTU-132 had amylase, CMCase, chitinase, pectinase, protease and lipase activities; S. thermophilus J57 had all enzyme activities except pectinase. Bacillus coagulans C45, B. licheniformis $\mathrm{A} 3$, and $\mathrm{S}$. thermophilus $\mathrm{J} 57$ manifested higher thermo-stable $\alpha$-amylase activities than $65.23 \mathrm{U} \mathrm{ml}^{-1}$ and $53 \mathrm{U} \mathrm{ml}^{-1}$ produced by Bacillus sp. PN5 (Saxena et al., 2007) and isolate Bacillus sp. YX-1 (Liu and Xu, 2008), respectively. All tested strains possessed higher CMCase activities than that of thermophilic Bacillus spp. (about $5.6 \mathrm{U} \mathrm{ml}^{-1}$ ) grown at $20-60{ }^{\circ} \mathrm{C}$ isolated from composts (Mayende et al., 2006). Bacillus coagulans C45, B. licheniformis $\mathrm{A} 3$, and $B$. smithii $\mathrm{F} 18$ had higher protease activities grown at $50^{\circ} \mathrm{C}$ than $25^{\circ} \mathrm{C}$ that was similar as the thermo-stable protease generated by thermophilic Bacillus sp., such as $B$. licheniformis and B. stearothermophilus (Haki and Rakshit, 2003). Therefore, all the tested isolates had much presumable potential for many industrial and biotechnical applications. Regarding to nitrogenase activity, B. coagulans $\mathrm{C} 45, B$. licheniformis $\mathrm{A} 3, \mathrm{~S}$. thermophilus J57, S. thermonitrificans NTU-88, A. fumigatus 04, and A. fumigatus NTU-132 had colony sizes of $2.4 \pm 0.2,4.0 \pm 0.3$, $1.7 \pm 0.5,8.2 \pm 0.2,26.2 \pm 2.1$, and $20.1 \pm 1.90 \mathrm{~mm}$ on Winogradsky's nitrogen-free mineral medium at $25^{\circ} \mathrm{C}$, respectively, while they had colony sizes of $5.0 \pm 1.1,13.8 \pm 3.8,4.9 \pm 1.2,21.3 \pm 1.3$, $5.1 \pm 0.3$, and $3.8 \pm 0.4 \mathrm{~mm}$ at $50^{\circ} \mathrm{C}$, respectively. Brevibacillus borstelensis SH168 formed swarming colonies at 25 and $50{ }^{\circ} \mathrm{C}$. Bacillus smithii F18 had a colony size of $3.8 \pm 0.6 \mathrm{~mm}$ at $50^{\circ} \mathrm{C}$. Thus, all the tested isolates had multiple enzyme activities grown at 25 and $50{ }^{\circ} \mathrm{C}$. 
Table 1

Enzyme activity indices of tested thermo-tolerant phosphate-solubilizing isolates after five days incubation

\begin{tabular}{|c|c|c|c|c|c|c|}
\hline Isolates & Amylase & CMCase & Chitinase & Pectinase & Protease & Lipase \\
\hline \multicolumn{7}{|c|}{ B. coagulans $\mathrm{C} 45$} \\
\hline $25^{\circ} \mathrm{C}$ & $1.11 \pm 0.07$ & $1.00 \pm 0.00$ & ND & $1.00 \pm 0.00$ & $1.54 \pm 0.05$ & $1.22 \pm 0.12$ \\
\hline $50^{\circ} \mathrm{C}$ & $1.32 \pm 0.15$ & $1.05 \pm 0.01$ & $1.04 \pm 0.01$ & $1.00 \pm 0.00$ & $1.09 \pm 0.07$ & $1.26 \pm 0.23$ \\
\hline \multicolumn{7}{|c|}{ B. licheniformis A3 } \\
\hline $25^{\circ} \mathrm{C}$ & $1.84 \pm 0.17$ & $2.76 \pm 0.33$ & $1.18 \pm 0.04$ & $1.00 \pm 0.00$ & $3.41 \pm 0.31$ & $1.78 \pm 0.04$ \\
\hline $50^{\circ} \mathrm{C}$ & $1.52 \pm 0.26$ & $2.31 \pm 0.50$ & $1.15 \pm 0.09$ & $1.00 \pm 0.00$ & $2.54 \pm 0.33$ & $1.11 \pm 0.02$ \\
\hline \multicolumn{7}{|c|}{ B. smithii $\mathrm{F} 18$} \\
\hline $25^{\circ} \mathrm{C}$ & $1.10 \pm 0.06$ & $1.00 \pm 0.00$ & ND & $1.00 \pm 0.00$ & ND & ND \\
\hline $50^{\circ} \mathrm{C}$ & $1.10 \pm 0.11$ & $1.19 \pm 0.04$ & $1.11 \pm 0.05$ & $1.00 \pm 0.00$ & $1.76 \pm 0.37$ & $1.10 \pm 0.04$ \\
\hline \multicolumn{7}{|c|}{ Bv. borstelensis SH168 } \\
\hline $25^{\circ} \mathrm{C}$ & $1.20 \pm 0.10$ & $1.05 \pm 0.02$ & $1.00 \pm 0.00$ & $1.00 \pm 0.00$ & $1.07 \pm 0.01$ & $1.61 \pm 0.32$ \\
\hline $50{ }^{\circ} \mathrm{C}$ & $1.20 \pm 0.03$ & $1.04 \pm 0.22$ & $1.00 \pm 0.00$ & $1.00 \pm 0.00$ & $1.07 \pm 0.08$ & $1.04 \pm 0.02$ \\
\hline \multicolumn{7}{|c|}{ S. thermophilus J57 } \\
\hline $25^{\circ} \mathrm{C}$ & $1.53 \pm 0.07$ & $1.00 \pm 0.00$ & $1.00 \pm 0.00$ & ND & $1.00 \pm 0.00$ & $1.44 \pm 0.15$ \\
\hline $50{ }^{\circ} \mathrm{C}$ & $1.88 \pm 0.31$ & $1.53 \pm 0.10$ & $1.00 \pm 0.00$ & ND & $1.10 \pm 0.06$ & $1.29 \pm 0.13$ \\
\hline \multicolumn{7}{|c|}{ S. thermonitrificans NTU-88 } \\
\hline $25^{\circ} \mathrm{C}$ & $1.43 \pm 0.17$ & $1.23 \pm 0.07$ & $1.00 \pm 0.00$ & $1.00 \pm 0.00$ & $1.13 \pm 0.09$ & $3.72 \pm 0.41$ \\
\hline $50^{\circ} \mathrm{C}$ & $1.24 \pm 0.14$ & $1.54 \pm 0.19$ & $1.00 \pm 0.00$ & $1.00 \pm 0.00$ & $1.13 \pm 0.07$ & $1.68 \pm 0.35$ \\
\hline \multicolumn{7}{|c|}{ A. fumigatus $\mathrm{O} 4$} \\
\hline $25^{\circ} \mathrm{C}$ & $1.05 \pm 0.04$ & $1.00 \pm 0.02$ & $1.10 \pm 0.03$ & $1.00 \pm 0.00$ & $1.11 \pm 0.00$ & $1.22 \pm 0.03$ \\
\hline $50^{\circ} \mathrm{C}$ & $1.82 \pm 0.26$ & $2.06 \pm 0.25$ & $1.27 \pm 0.13$ & $1.00 \pm 0.00$ & $1.09 \pm 0.03$ & $1.97 \pm 0.35$ \\
\hline \multicolumn{7}{|c|}{ A. fumigatus NTU-132 } \\
\hline $25^{\circ} \mathrm{C}$ & $1.09 \pm 0.04$ & $1.05 \pm 0.00$ & $1.03 \pm 0.03$ & $1.00 \pm 0.00$ & $1.15 \pm 0.05$ & $1.34 \pm 0.06$ \\
\hline $50^{\circ} \mathrm{C}$ & $1.63 \pm 0.20$ & $1.74 \pm 0.27$ & $1.20 \pm 0.10$ & $1.00 \pm 0.00$ & $1.16 \pm 0.11$ & $1.74 \pm 0.29$ \\
\hline
\end{tabular}

The enzyme activity index is the halo ratio of clear zone/colony size of tested isolates.

Data are the means \pm SD $(n \geqslant 3)$. ND: Not determined because the strain did not grow very well.

Table 2

Enzyme activities of tested thermo-tolerant phosphate-solubilizing isolates

\begin{tabular}{|c|c|c|c|}
\hline Isolates & $\alpha$-Amylase $\left(\mathrm{U} \mathrm{ml}^{-1}\right)^{\mathrm{a}}$ & CMCase $\left(\mathrm{U} \mathrm{ml}^{-1}\right)^{\mathrm{a}}$ & Protease $\left(\mathrm{U} \mathrm{ml}^{-1}\right)^{\mathrm{b}}$ \\
\hline \multicolumn{4}{|c|}{ B. coagulans C45 } \\
\hline $25^{\circ} \mathrm{C}$ & $50.2 \pm 8.6$ & $35.5 \pm 0.8$ & $405.2 \pm 50.2$ \\
\hline $50{ }^{\circ} \mathrm{C}$ & $79.6 \pm 5.5$ & $77.5 \pm 6.9$ & $982.5 \pm 10.4$ \\
\hline \multicolumn{4}{|c|}{ B. licheniformis A3 } \\
\hline $25^{\circ} \mathrm{C}$ & $68.2 \pm 8.9$ & $58.2 \pm 2.8$ & $805.2 \pm 90.2$ \\
\hline $50^{\circ} \mathrm{C}$ & $99.5 \pm 8.5$ & $97.2 \pm 9.5$ & $1102.2 \pm 50.2$ \\
\hline \multicolumn{4}{|c|}{ B. smithii F18 } \\
\hline $25^{\circ} \mathrm{C}$ & $15.2 \pm 0.2$ & $10.2 \pm 1.5$ & $60.2 \pm 10.2$ \\
\hline $50{ }^{\circ} \mathrm{C}$ & $62.1 \pm 8.2$ & $52.2 \pm 5.8$ & $888.2 \pm 75.4$ \\
\hline \multicolumn{4}{|c|}{ S. thermophilus J57 } \\
\hline $25^{\circ} \mathrm{C}$ & $67.2 \pm 7.5$ & $20.2 \pm 2.9$ & $352.2 \pm 58.2$ \\
\hline $50^{\circ} \mathrm{C}$ & $97.2 \pm 8.5$ & $98.2 \pm 6.9$ & $452.2 \pm 38.2$ \\
\hline \multicolumn{4}{|c|}{ A. fumigatus $\mathrm{O} 4$} \\
\hline $25^{\circ} \mathrm{C}$ & $79.9 \pm 6.9$ & $90.1 \pm 8.2$ & $1002.5 \pm 20.5$ \\
\hline $50{ }^{\circ} \mathrm{C}$ & $15.0 \pm 0.9$ & $25.0 \pm 2.8$ & $258.5 \pm 64.5$ \\
\hline
\end{tabular}

Data are the means $\pm \operatorname{SD}(n \geqslant 3)$.

a One unit (U) of enzyme activity is defined as the amount of enzyme for producing $1 \mu \mathrm{mol} \mathrm{ml} l^{-1}$ of glucose per minute.

${ }^{b}$ One unit (U) of protease activity is defined as the amount of enzyme for liberating $1 \mu \mathrm{g} \mathrm{ml}^{-1}$ of tyrosine per minute.

\subsection{Inorganic phosphate-solubilizing activity}

The animal wastes contained substantial amounts of phosphorus. Sixty to ninety percent of the phosphorus in animal waste is in inorganic forms and $10-40 \%$ is in organic forms (Barnett, 1994). Therefore, the $\mathrm{Ca}_{3}\left(\mathrm{PO}_{4}\right)_{2}, \mathrm{AlPO}_{4}, \mathrm{FePO}_{4}$, hydroxyapatite (HA), and rock phosphate (RP)-solubilizing activities of the isolates were determined, and the phosphate-solubilizing activities are shown in Tables 3 and 4. Bacillus coagulans C45, B. smithii F18, A. fumigatus 04 , and $A$. fumigatus NTU-132 could solubilize all phosphate substances at 25 and $50{ }^{\circ} \mathrm{C}$. Bacillus licheniformis A3 and $S$. thermophilus J57 could solubilize HA; while Bv. borstelensis SH168 and $S$. thermonitrificans NTU-88 could solubilize $\mathrm{AlPO}_{4}$ at 25 and $50{ }^{\circ} \mathrm{C}$.

Bacillus smithii $\mathrm{F} 18$ had the highest $\mathrm{Ca}_{3}\left(\mathrm{PO}_{4}\right)_{2^{-}}, \mathrm{AlPO}_{4^{-}} \mathrm{FePO}_{4^{-}}$, and RP-solubilizing activities along with the lowest $\mathrm{pH}$ in broth at $50{ }^{\circ} \mathrm{C}$; followed by B. coagulans $\mathrm{C} 45$ (Table 4). However, A. fumigatus $\mathrm{O} 4$ and $A$. fumigatus NTU-132 had high $\mathrm{AlPO}_{4}$ - and $\mathrm{FePO}_{4}$-solubilizing activities along with appropriate low pH value at $25^{\circ} \mathrm{C} ; B$. coagulans $\mathrm{C} 45$ had high $\mathrm{Ca}_{3}\left(\mathrm{PO}_{4}\right)_{2}$-solubilizing activity, and $S$. thermophilus J57 had high RP-solubilizing activity along with low $\mathrm{pH}$. Bacillus coagulans C45, B. licheniformis A3, B. smithii F18, Bv. borstelensis SH168, and S. thermonitrificans NTU-88 had higher phosphate-solubilizing activities at $50{ }^{\circ} \mathrm{C}$ than those at $25^{\circ} \mathrm{C}$; while $S$. thermophilus $\mathrm{J} 57$, A. fumigatus $\mathrm{O} 4$ and A. fumigatus NTU-132 had higher activities at $25{ }^{\circ} \mathrm{C}$ than those at $50{ }^{\circ} \mathrm{C}$. Therefore, the former isolates are thermo-tolerant phosphate-solubilizing microbes and the latter are mesophilic phosphate-solubilizing microbes. Bacillus coagulans C45 had high phosphate-solubilizing activity at both 25 and $50{ }^{\circ} \mathrm{C}$ and appeared to be the best candidate phosphate-solubilizer for enhancing soluble phosphorus concentration during biofertilizer preparation. There is no definite correlation between PSAIs with plate assay and phosphate-solubilizing activities with broth assay in thermo-tolerant phosphate-solubilizing microbes. Nautiyal (1999) reported that the phosphate-solubilizing bacteria Pseudomonas aerogenes could not produce halo when it grew on PVK or NBRIP plate; but it could solubilize 14 and $31 \mu \mathrm{g} \mathrm{m}^{-1}$ of phosphorus when it grew in PVK and NBRIP broth, respectively. Pseudomonas sp. 1 also could not produce halo on PVK plate; but it could mobilize $8 \mu \mathrm{g} \mathrm{ml}^{-1}$ of phosphorus in PVK broth. Similar phenomena were also found in this study. Bacillus smithii F18 growing at $25^{\circ} \mathrm{C}$ had small halos, but it could solubilize five kinds of phosphate substrates; S. thermophilus $\mathrm{J} 57$ and A. fumigatus 04 also had small clear zones and PSAIs at 25 and $50{ }^{\circ} \mathrm{C}$, but they could manifest significantly $(P<0.001)$ higher phosphate-solubilizing activities at $25^{\circ} \mathrm{C}$ than those at $50{ }^{\circ} \mathrm{C}$ (Tables 3 and 4 ).

\subsection{Biofertilizer preparation}

During biofertilizer preparation, microbes decomposed the organic matter and released the fermentation heat (Yang, 2003). The biofertilizer temperatures increased rapidly during composting, peaking on day $7(P<0.001)$, and then decreased gradually for the maturation of biofertilizer (Fig. 1). The temperature patterns were similar to the commercial composting processes (Pai 
Table 3

Phosphate-solubilizing activity indices of tested thermo-tolerant phosphate-solubilizing isolates for five days incubation

\begin{tabular}{|c|c|c|c|c|c|}
\hline Isolates & PVK Medium & $\mathrm{AlPO}_{4}$ Medium & $\mathrm{FePO}_{4}$ Medium & HA Medium & RP Medium \\
\hline \multicolumn{6}{|c|}{ B. coagulans $\mathrm{C} 45$} \\
\hline $25^{\circ} \mathrm{C}$ & $1.09 \pm 0.00$ & $1.10 \pm 0.03$ & $1.00 \pm 0.00$ & $1.80 \pm 0.43$ & $1.10 \pm 0.04$ \\
\hline $50{ }^{\circ} \mathrm{C}$ & $2.45 \pm 0.25$ & $1.11 \pm 0.01$ & $1.38 \pm 0.13$ & $2.06 \pm 0.15$ & $1.10 \pm 0.08$ \\
\hline \multicolumn{6}{|c|}{ B. licheniformis A3 } \\
\hline $25^{\circ} \mathrm{C}$ & $1.54 \pm 0.02$ & ND & ND & $1.10 \pm 0.04$ & $1.12 \pm 0.05$ \\
\hline $50{ }^{\circ} \mathrm{C}$ & $1.96 \pm 0.23$ & ND & ND & $1.12 \pm 0.04$ & $1.12 \pm 0.01$ \\
\hline \multicolumn{6}{|c|}{ B. smithii F18 } \\
\hline $25^{\circ} \mathrm{C}$ & $1.10 \pm 0.01$ & $1.10 \pm 0.03$ & $1.00 \pm 0.00$ & $1.00 \pm 0.00$ & $1.00 \pm 0.00$ \\
\hline $50^{\circ} \mathrm{C}$ & $1.36 \pm 0.08$ & $1.12 \pm 0.06$ & $1.24 \pm 0.02$ & $1.17 \pm 0.06$ & $1.06 \pm 0.01$ \\
\hline \multicolumn{6}{|c|}{ Bv. borstelensis SH168 } \\
\hline $25^{\circ} \mathrm{C}$ & $1.08 \pm 0.00$ & $1.08 \pm 0.03$ & ND & ND & $1.07 \pm 0.05$ \\
\hline $50^{\circ} \mathrm{C}$ & $1.09 \pm 0.04$ & $1.09 \pm 0.07$ & ND & ND & $1.07 \pm 0.02$ \\
\hline \multicolumn{6}{|c|}{ S. thermophilus J57 } \\
\hline $25^{\circ} \mathrm{C}$ & $1.08 \pm 0.03$ & ND & ND & ND & $1.07 \pm 0.02$ \\
\hline $50^{\circ} \mathrm{C}$ & $1.00 \pm 0.00$ & ND & ND & $1.10 \pm 0.01$ & $1.05 \pm 0.02$ \\
\hline \multicolumn{6}{|c|}{ S. thermonitrificans NTU-88 } \\
\hline $25^{\circ} \mathrm{C}$ & $1.04 \pm 0.01$ & $1.06 \pm 0.03$ & ND & ND & $1.05 \pm 0.03$ \\
\hline $50^{\circ} \mathrm{C}$ & $1.05 \pm 0.02$ & $1.06 \pm 0.02$ & ND & ND & $1.06 \pm 0.03$ \\
\hline \multicolumn{6}{|c|}{ A. fumigatus $\mathrm{O} 4$} \\
\hline $25^{\circ} \mathrm{C}$ & $1.06 \pm 0.02$ & $1.11 \pm 0.02$ & $1.07 \pm 0.02$ & $1.08 \pm 0.04$ & $1.07 \pm 0.05$ \\
\hline $50{ }^{\circ} \mathrm{C}$ & $1.04 \pm 0.01$ & $1.02 \pm 0.01$ & $1.07 \pm 0.01$ & $1.07 \pm 0.04$ & $1.07 \pm 0.06$ \\
\hline \multicolumn{6}{|c|}{ A. fumigatus NTU-132 } \\
\hline $25^{\circ} \mathrm{C}$ & $1.08 \pm 0.03$ & $1.23 \pm 0.07$ & $1.02 \pm 0.01$ & $1.08 \pm 0.04$ & $1.09 \pm 0.05$ \\
\hline $50^{\circ} \mathrm{C}$ & $1.08 \pm 0.02$ & $1.20 \pm 0.11$ & $1.03 \pm 0.02$ & $1.06 \pm 0.01$ & $1.05 \pm 0.02$ \\
\hline
\end{tabular}

The phosphate-solubilizing activity index is the halo ratio of clear zone/ colony size of tested isolates.

Data are the means \pm SD $(n \geqslant 3)$. ND: Not determined because the strain did not grow very well.

Table 4

Phosphate-solubilizing activity and $\mathrm{pH}$ of tested thermo-tolerant phosphate-solubilizing isolates

\begin{tabular}{|c|c|c|c|c|}
\hline Isolates & PVK Broth & $\mathrm{AlPO}_{4}$ Broth & $\mathrm{FePO}_{4}$ Broth & RP Broth \\
\hline \multicolumn{5}{|l|}{ B. coagulans $\mathrm{C} 45$} \\
\hline $25^{\circ} \mathrm{C}$ soluble $\mathrm{P}\left(\mu \mathrm{g} \mathrm{ml}^{-1}\right)$ & $370.2 \pm 16.0$ & $9.5 \pm 0.3$ & $6.4 \pm 0.7$ & $95.2 \pm 10.5$ \\
\hline $\mathrm{pH}$ & $4.4 \pm 0.1$ & $5.8 \pm 0.1$ & $6.2 \pm 0.1$ & $5.0 \pm 0.1$ \\
\hline $50^{\circ} \mathrm{C}$ soluble $\mathrm{P}\left(\mu \mathrm{g} \mathrm{ml}^{-1}\right)$ & $466.8 \pm 21.2$ & $77.5 \pm 6.9$ & $8.3 \pm 0.4$ & $171.2 \pm 13.2$ \\
\hline $\mathrm{pH}$ & $4.0 \pm 0.1$ & $5.0 \pm 0.1$ & $5.9 \pm 0.1$ & $4.8 \pm 0.1$ \\
\hline \multicolumn{5}{|l|}{ B. licheniformis A3 } \\
\hline $25^{\circ} \mathrm{C}$ soluble $\mathrm{P}\left(\mu \mathrm{g} \mathrm{ml}^{-1}\right)$ & $58.4 \pm 6.5$ & ND & ND & $14.8 \pm 1.5$ \\
\hline $\mathrm{pH}$ & $4.8 \pm 0.1$ & ND & ND & $5.2 \pm 0.1$ \\
\hline $50{ }^{\circ} \mathrm{C}$ soluble $\mathrm{P}\left(\mu \mathrm{g} \mathrm{ml}^{-1}\right)$ & $80.2 \pm 3.3$ & ND & ND & $18.2 \pm 1.0$ \\
\hline $\mathrm{pH}$ & $5.1 \pm 0.0$ & ND & ND & $5.5 \pm 0.0$ \\
\hline \multicolumn{5}{|l|}{ B. smithii F18 } \\
\hline $25^{\circ} \mathrm{C}$ soluble $\mathrm{P}\left(\mu \mathrm{g} \mathrm{ml}^{-1}\right)$ & $7.3 \pm 0.3$ & $6.6 \pm 0.3$ & $3.6 \pm 0.2$ & $7.3 \pm 1.1$ \\
\hline $\mathrm{pH}$ & $6.0 \pm 0.0$ & $6.2 \pm 0.0$ & $6.4 \pm 0.1$ & $6.1 \pm 0.0$ \\
\hline $50{ }^{\circ} \mathrm{C}$ soluble $\mathrm{P}\left(\mu \mathrm{g} \mathrm{ml}^{-1}\right)$ & $544.2 \pm 30.2$ & $119.9 \pm 3.1$ & $10.2 \pm 0.4$ & $451.1 \pm 24.9$ \\
\hline $\mathrm{pH}$ & $4.0 \pm 0.0$ & $5.1 \pm 0.1$ & $5.8 \pm 0.1$ & $4.8 \pm 0.1$ \\
\hline \multicolumn{5}{|l|}{ Bv. borstelensis SH168 } \\
\hline $25^{\circ} \mathrm{C}$ soluble $\mathrm{P}\left(\mu \mathrm{g} \mathrm{ml}^{-1}\right)$ & $172.5 \pm 11.5$ & $5.6 \pm 0.2$ & ND & $10.9 \pm 1.4$ \\
\hline $\mathrm{pH}$ & $5.0 \pm 0.0$ & $6.3 \pm 0.0$ & ND & $5.5 \pm 0.1$ \\
\hline $50{ }^{\circ} \mathrm{C}$ soluble $\mathrm{P}\left(\mu \mathrm{g} \mathrm{ml}^{-1}\right)$ & $205.6 \pm 19.9$ & $37.0 \pm 2.5$ & ND & $102.1 \pm 13.0$ \\
\hline $\mathrm{pH}$ & $4.5 \pm 0.0$ & $5.3 \pm 0.1$ & ND & $5.1 \pm 0.0$ \\
\hline \multicolumn{5}{|l|}{ S. thermophilus J57 } \\
\hline $25^{\circ} \mathrm{C}$ soluble $\mathrm{P}\left(\mu \mathrm{g} \mathrm{ml}^{-1}\right)$ & $111.7 \pm 9.2$ & ND & ND & $288.1 \pm 38.6$ \\
\hline $\mathrm{pH}$ & $5.1 \pm 0.0$ & ND & ND & $4.8 \pm 0.1$ \\
\hline $50{ }^{\circ} \mathrm{C}$ soluble $\mathrm{P}\left(\mu \mathrm{g} \mathrm{ml}^{-1}\right)$ & $78.2 \pm 7.3$ & ND & ND & $190.2 \pm 17.6$ \\
\hline $\mathrm{pH}$ & $5.2 \pm 0.1$ & ND & ND & $5.0 \pm 0.0$ \\
\hline \multicolumn{5}{|l|}{ S. thermonitrificans NTU-88 } \\
\hline $25^{\circ} \mathrm{C}$ soluble $\mathrm{P}\left(\mu \mathrm{g} \mathrm{ml}^{-1}\right)$ & $4.1 \pm 0.3$ & $6.2 \pm 0.7$ & ND & $6.9 \pm 0.9$ \\
\hline $\mathrm{pH}$ & $6.7 \pm 0.0$ & $6.1 \pm 0.0$ & ND & $5.9 \pm 0.1$ \\
\hline $50{ }^{\circ} \mathrm{C}$ soluble $\mathrm{P}\left(\mu \mathrm{g} \mathrm{ml}^{-1}\right)$ & $57.0 \pm 2.7$ & $9.4 \pm 1.0$ & ND & $10.2 \pm 1.4$ \\
\hline $\mathrm{pH}$ & $5.2 \pm 0.0$ & $5.8 \pm 0.0$ & ND & $5.6 \pm 0.1$ \\
\hline \multicolumn{5}{|l|}{ A. fumigatus $\mathrm{O} 4$} \\
\hline $25^{\circ} \mathrm{C}$ soluble $\mathrm{P}\left(\mu \mathrm{g} \mathrm{ml}^{-1}\right)$ & $113.1 \pm 15.1$ & $106.2 \pm 7.9$ & $92.4 \pm 5.8$ & $130.4 \pm 13.4$ \\
\hline $\mathrm{pH}$ & $5.2 \pm 0.1$ & $5.2 \pm 0.0$ & $5.5 \pm 0.0$ & $5.1 \pm 0.1$ \\
\hline $50{ }^{\circ} \mathrm{C}$ soluble $\mathrm{P}\left(\mu \mathrm{g} \mathrm{ml}^{-1}\right)$ & $11.6 \pm 2.3$ & $6.5 \pm 0.6$ & $5.4 \pm 1.0$ & $11.5 \pm 1.1$ \\
\hline $\mathrm{pH}$ & $5.2 \pm 0.0$ & $6.0 \pm 0.0$ & $6.1 \pm 0.1$ & $6.0 \pm 0.1$ \\
\hline \multicolumn{5}{|l|}{ A. fumigatus NTU-132 } \\
\hline $25^{\circ} \mathrm{C}$ soluble $\mathrm{P}\left(\mu \mathrm{g} \mathrm{ml}^{-1}\right)$ & $12.6 \pm 1.2$ & $22.4 \pm 3.2$ & $14.2 \pm 0.7$ & $45.7 \pm 4.5$ \\
\hline $\mathrm{pH}$ & $5.6 \pm 0.0$ & $5.5 \pm 0.1$ & $5.7 \pm 0.1$ & $5.5 \pm 0.1$ \\
\hline $50{ }^{\circ} \mathrm{C}$ soluble $\mathrm{P}\left(\mu \mathrm{g} \mathrm{ml}^{-1}\right)$ & $7.0 \pm 1.1$ & $6.4 \pm 0.7$ & $2.6 \pm 0.3$ & $6.6 \pm 0.5$ \\
\hline $\mathrm{pH}$ & $5.9 \pm 0.0$ & $6.2 \pm 0.0$ & $6.5 \pm 0.0$ & $6.1 \pm 0.1$ \\
\hline
\end{tabular}

Data are the means \pm SD $(n \geqslant 3)$. ND: Not determined because the strain did not grow very well. 


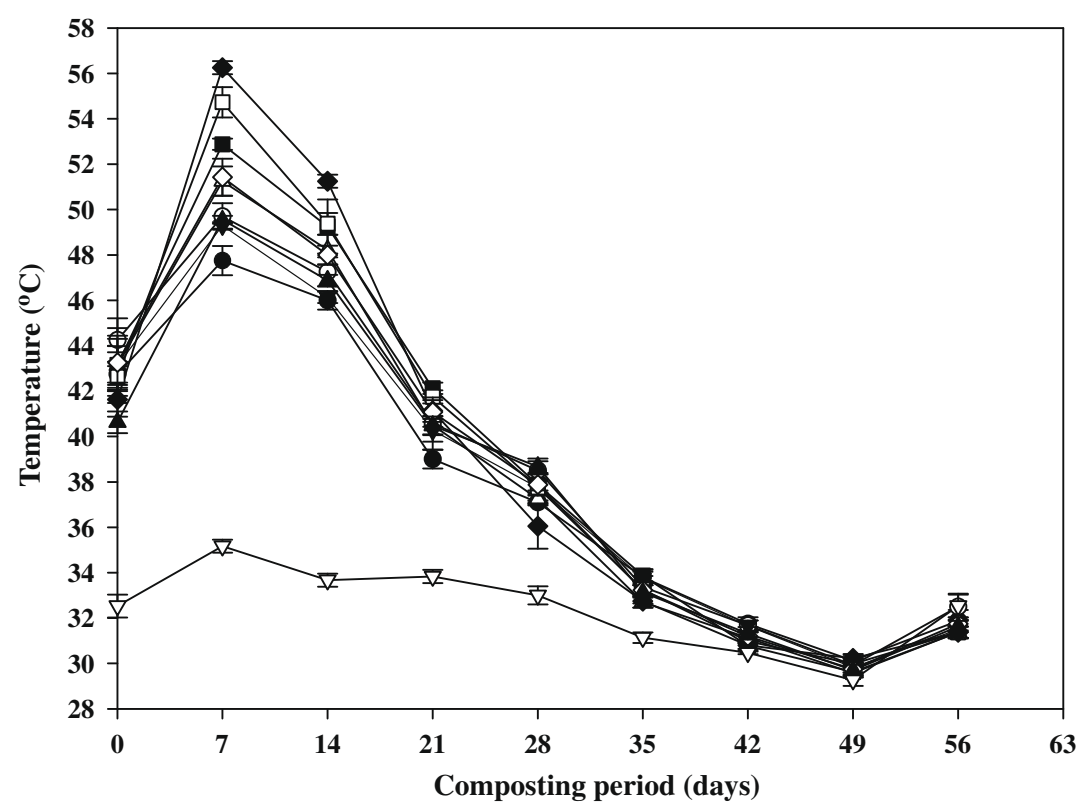

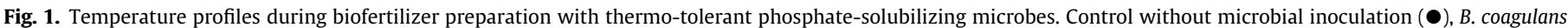

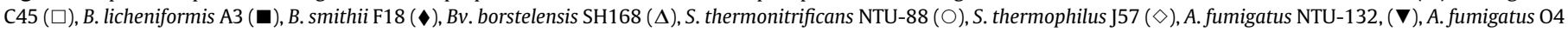
$(\Lambda)$, and air temperatures $(\nabla)$. Data points are means and vertical bars are standard deviations $(n \geqslant 3)$.

et al., 2003), preparation of biofertilizers treated with polyelectrolyte oxygen detoxifier (Chang and Yang, 2006), and preparation of food waste biofertilizers (Tsai et al., 2007). Compost inoculated with $B$. smithii F18 had the significantly $(P<0.01)$ highest temperature, followed by $B$. coagulans $\mathrm{C} 45$, B. licheniformis A3, Bv. borstelensis SH168, S. thermophilus J57, S. thermonitrificans NTU-88, A. fumigatus 04 , A. fumigatus NTU-132, and the non-inoculated control compost had the lowest. Moisture content decreased gradually during composting due to the vaporization in high temperature (Chang and Yang, 2006). Moisture content of compost inoculated with $B$. smithii F18 decreased faster along with the higher temperature, while that of the non-inoculated control compost decreased less for the lower temperature. The final moisture content of compost inoculated with $S$. thermonitrificans NTU-88 was the lowest $(42.7 \pm 1.9 \%)$, while the moisture content of control compost without inoculation manifested the highest $(44.2 \pm 0.8 \%)$. Therefore, inoculation with thermo-tolerant phosphate-solubilizing microbes stimulated the organic matter decomposition, increased the temperature, and decreased the moisture content of biofertilizer. Same phenomenon has also found in open field composting (Pai et al., 2003).

The $\mathrm{pH}$ of composts increased gradually over time to a slightly alkali $\mathrm{pH}$ after 56 days composting due to the degradation of nitrogen-containing materials, the formation of $\mathrm{NH}_{4}^{+}$ions and the release of $\mathrm{OH}^{-}$by hydrolysis. The compost inoculated with $B$. smithii $\mathrm{F} 18$ had the highest $\mathrm{pH}(8.8 \pm 0.1)$, followed by $B$. coagulans C45 (8.7 \pm 0.1$)$ and $B$. licheniformis A3 $(8.7 \pm 0.1)$; the control compost without inoculation had the lowest $\mathrm{pH}(8.3 \pm 0.0)$. The slight alkaline $\mathrm{pH}$ of biofertilizer is benefit in agriculture because of this contributes to the neutralization the acidic agricultural soil (Huang, 1991; Fageria and Baligar, 2001).

The ash content increased significantly during preparation since the organic materials were decomposed to form the metabolic gases, such as $\mathrm{CO}_{2}, \mathrm{H}_{2} \mathrm{O}$, or $\mathrm{N}_{2} \mathrm{O}$ (Yang and Chen, 2003; Chang and Yang, 2006). Compost inoculated with B. smithii F18 had the highest ash content $(52.8 \pm 2.1 \%)$, followed by B. coagulans C45 $(51.8 \pm 1.1 \%)$ and $B$. licheniformis $A 3(50.8 \pm 2.1 \%)$, and the noninoculated control compost had the lowest $(46.9 \pm 1.9 \%)$.
Total organic carbon decreased significantly during composting due to the degradation of organic matter (Chang and Yang, 2006). Compost inoculated with $B$. smithii F18 possessed the lowest final total organic carbon content ( $21.6 \pm 1.1 \%)$, followed by $B$. coagulans C45 $(22.5 \pm 0.7 \%)$ and $B$. licheniformis A3 (23.4 $\pm 1.3 \%)$; the control compost without inoculation manifested the highest $(27.9 \pm$ $2.1 \%)$. Total nitrogen increased throughout preparation. Compost inoculated with B. smithii F18 and B. coagulans $\mathrm{C} 45$ had the highest total nitrogen content $(1.95 \pm 0.01 \%)$, and the non-inoculated control compost had the lowest $(1.90 \pm 0.01 \%)$. The $\mathrm{C} / \mathrm{N}$ ratios decreased significantly during composting. Compost inoculated with B. smithii F18 presented the lowest final $C / N$ ratio $(11.1 \pm 0.6)$, followed by B. coagulans C45 (11.5 \pm 0.4$)$, B. licheniformis A3 (12.1 \pm $0.8), \quad B v$. borstelensis SH168 (12.8 \pm 0.8$), \quad S$. thermophilus J57 $(12.9 \pm 1.3)$, S. thermonitrificans NTU-88 $(13.0 \pm 1.2)$, A. fumigatus O4 (13.1 \pm 1.1$)$, A. fumigatus NTU-132 (13.4 \pm 0.7$)$; the control compost without inoculation showed the highest $\mathrm{C} / \mathrm{N}$ ratio $(14.6 \pm 1.0)$. Therefore, compost inoculated with thermo-tolerant phosphatesolubilizing microbes can accelerate the decomposition of agricultural and animal wastes that is similar with the results reported by Yang and Chen (2003). Bacteria decomposed the wastes faster than those of actinomycetes and fungi.

The germination rate of alfalfa seed increased significantly during first 35 days composting, and then remained steady till day 56. The composts inoculated with $B$. smithii F18 and B. coagulans C45 manifested germination rates of $92.0 \pm 4.0$ and $90.7 \pm 2.3 \%$ for 14 days composting, respectively, but those of the other treatments were below $90 \%$. After 21 days composting, the germination rates persisted above $90 \%$ throughout till day 56. Inoculation with thermo-tolerant phosphate-solubilizing microbes increased the germination rate of alfalfa seed. During composting, organic matter is degraded, and phytotoxic compounds that inhibit seed germination, such as ammonia, ethylene oxide, acetic acid, propanoic acid, and $n$-butyric acid, are removed and enhanced the germination rate (Yang and Chen, 2003).

Dry weight loss increased significantly during preparation due to the production of metabolic gases, such as $\mathrm{CO}_{2}$ and $\mathrm{N}_{2} \mathrm{O}$ (Chang and Yang, 2006). Compost inoculated with B. smithii $\mathrm{F} 18$ expressed 
the highest dry weight loss ( $40.9 \pm 0.3 \%$ ), followed by $B$. coagulans C45 $(40.2 \pm 4.8 \%)$ and B. licheniformis A3 $(33.2 \pm 0.7 \%)$; the control compost without inoculation had the significantly $(P<0.001)$ lowest dry weight loss $(29.3 \pm 1.3 \%)$.

The color and odor of biofertilizers during preparation initially were grayish brown, pungent, and smelled strongly of ammonia. Because the humic substance content rose gradually during composting, the colors of the compost became dark brown (Yang and Chen, 2003). Over 21 days composting, the color of compost inoculated with B. coagulans C45, B. licheniformis A3, B. smithii F18, Bv. borstelensis SH168, and S. thermophilus J57 changed from grayish brown to deeply brown. Similar color transformations took place over 28 days in the compost inoculated with $S$. thermonitrificans NTU-88, A. fumigatus 04, and A. fumigatus NTU-132, and in the non-inoculated control compost. After 49 days of composting, the composts inoculated with thermo-tolerant phosphate-solubilizing microbes smelled fragrant, earthy, and generally "good". It has been reported that rice straw and hog waste composts inoculated with S. thermonitrificans NTU-88, Streptococcus sp. NTU-130, A. fumigatus NTU-132, and mixed cultures of these three strains reduced the odors and improved the smell to earthy or odorless (Yang and Chen, 2003). Similarly, it had been reported that the odor of volatile fatty acids was removed with the inoculations of thermophilic Streptomyces sp. No. 101, Thermoactinomyces sp. No. 64, and Micromonospora sp. No. 604 during cereal shochu-distillery waste composting (Tanaka et al., 1995). Thus, inoculating raw waste materials with the tested strains can reduce the odor and improve the smell associated with composting.

\subsection{Phosphorus content}

Total phosphorus content increased significantly during composting due to the decomposition of organic waste through the loss of dry weight (Fig. 2a). Compost inoculated with B. smithii F18 had the highest total phosphorus content of $56.3 \pm 1.0 \mathrm{~g} \mathrm{~kg}^{-1}$, followed by $B$. coagulans $C 45\left(56.2 \pm 1.3 \mathrm{~g} \mathrm{~kg}^{-1}\right)$; the control compost without inoculation had the lowest $\left(49.2 \pm 1.4 \mathrm{~g} \mathrm{~kg}^{-1}\right)$. The soluble phosphorus content decreased significantly by day 7

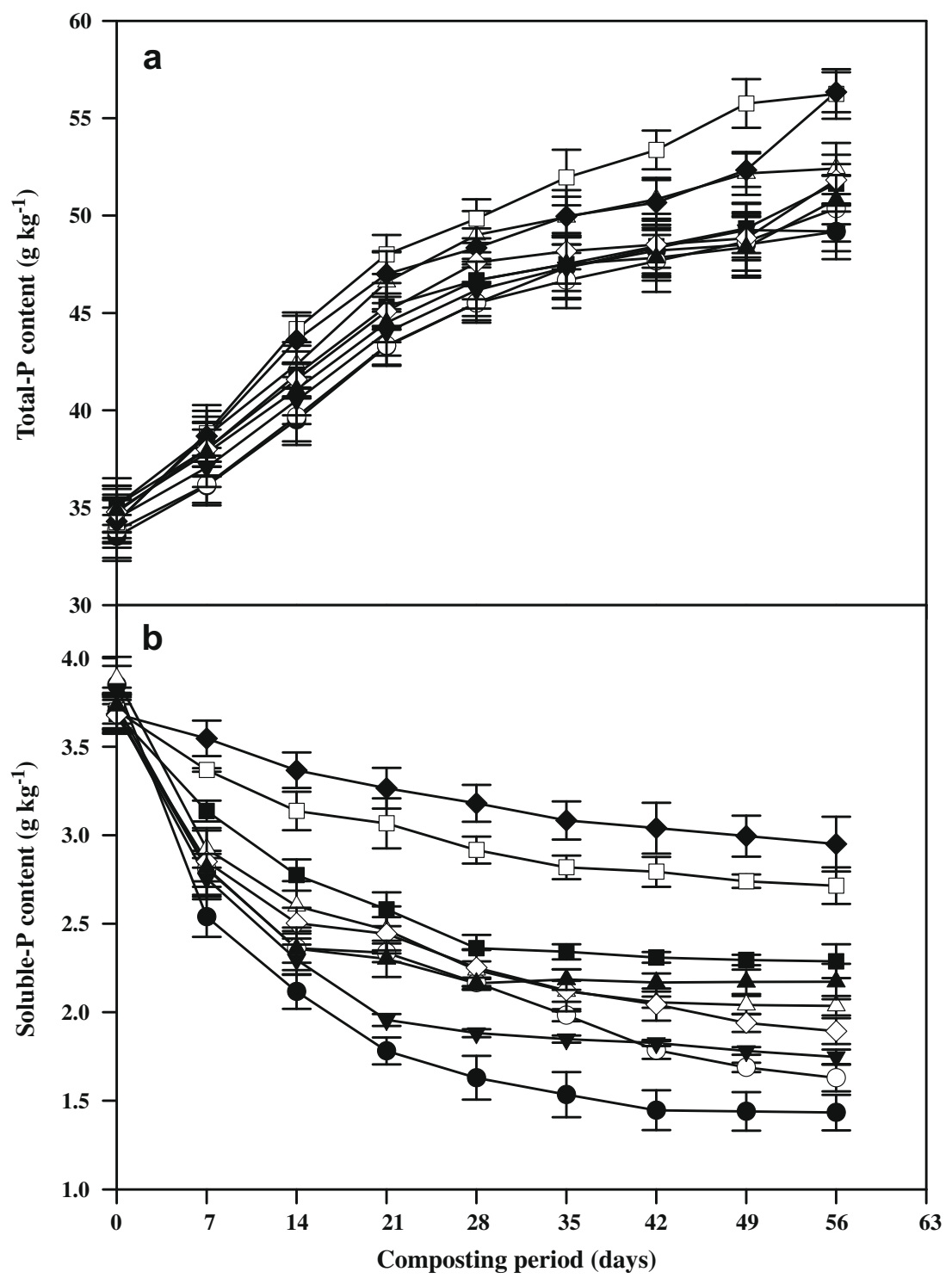

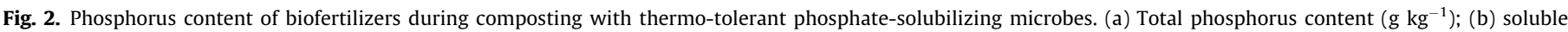

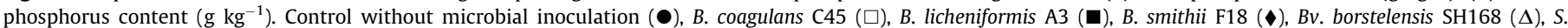

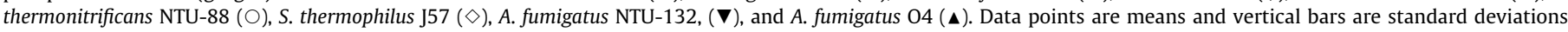
$(n \geqslant 3)$. 
$\left(2.5 \pm 0.1-3.5 \pm 0.1 \mathrm{~g} \mathrm{~kg}^{-1}\right)$, then dropped gradually and ultimately stabilized at $1.4 \pm 0.1-3.0 \pm 0.2 \mathrm{~g} \mathrm{~kg}^{-1}$ after 56 days composting (Fig. 2b). The soluble phosphorus content of compost inoculated with $B$. smithii $\mathrm{F} 18$ had the highest, followed by $B$. coagulans $C 45$, B. licheniformis A3, A. fumigatus O4, Bv. borstelensis SH168, S. thermophilus J57, A. fumigatus NTU-132, and S. thermonitrificans NTU88; the control compost without inoculation had the lowest soluble phosphorus content. The microbial activities and populations amplified more vigorously at early composting period resulting in the consumption of soluble phosphorus for microbial growth. Inoculation of thermo-tolerant phosphate-solubilizing microbes can tolerant high temperature and solubilize phosphate to supply microbial growth and increase the soluble phosphorus content in composts (Chang and Yang, 2006).

Initially, the percentage of soluble phosphorus in the total phosphorus was $10.6 \pm 1.3-11.5 \pm 1.0 \%$, and the value decreased significantly $(P<0.001)$ to $7.1 \pm 0.6-9.2 \pm 0.8 \%$ by day 7 for the microbial growth and decreased gradually. After 56 days of composting, the compost inoculated with $B$. smithii F18 had the highest percentage $(5.3 \pm 0.6 \%)$ of soluble phosphorus in the total phosphorus of composts, followed by B. coagulans C45 (4.9 $\pm 0.7 \%)$, B. licheniformis A3 ( $4.5 \pm 0.6 \%)$, A. fumigatus $\mathrm{O} 4(4.3 \pm 0.7 \%), B v$. borstelensis SH168 $(3.9 \pm 0.4 \%), S$. thermophilus J57 (3.7 $\pm 0.2 \%)$, A. fumigatus NTU-132 (3.6 $\pm 0.1 \%)$, and $S$. thermonitrificans NTU-88 (3.2 $\pm 0.3 \%)$; the control non-inoculated compost had the lowest $(2.9 \pm 0.2 \%)$. These results indicate that inoculation with thermo-tolerant phosphatesolubilizing microbes can increase the soluble phosphorus content. Bacteria solubilize phosphate more effectively than do actinomycetes and fungi. Similar phenomena were also reported by Zayed and Abdel-Motaal (2005a,b) and Biswas and Narayanasamy (2006).

\subsection{Microbial population}

During composting, the populations of mesophilic and thermotolerant bacteria, phosphate-solubilizing bacteria and proteolytic microbes increased rapidly to reach their peaks on day 14 com-

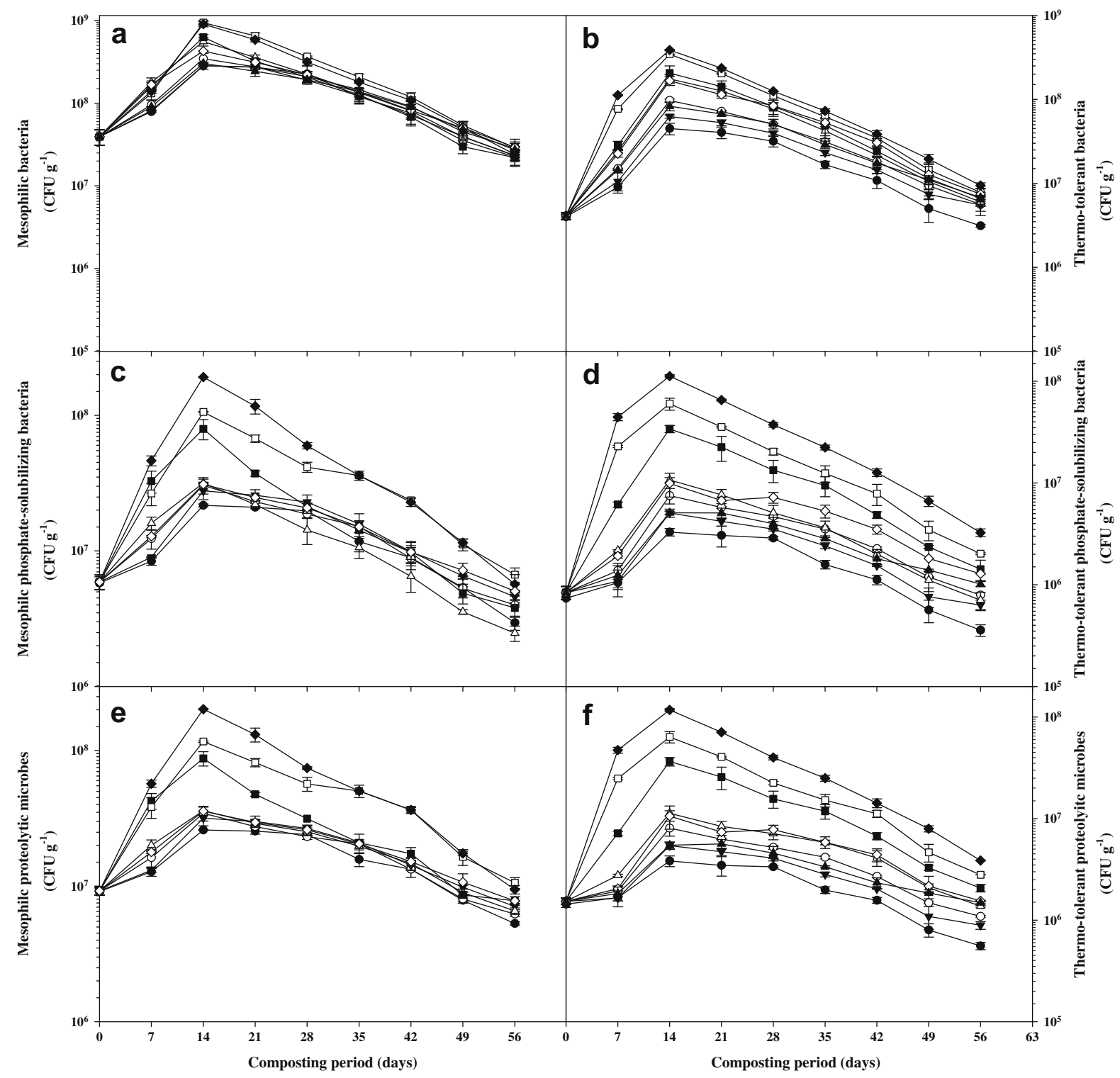

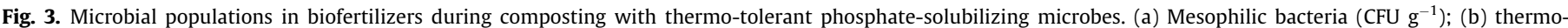

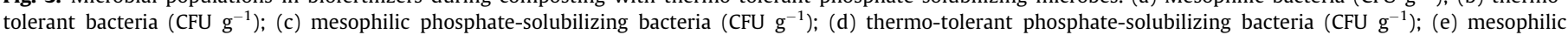

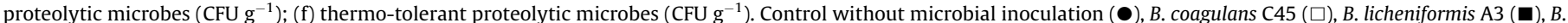

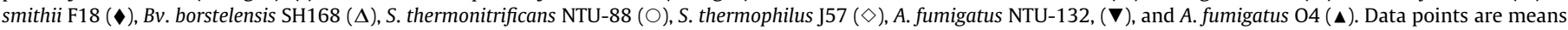
and vertical bars are standard deviations $(n \geqslant 3)$. 
posting and then decreased gradually for the maturation of biofertilizer (Fig. 3). Compost inoculated with B. smithii F18 had the largest populations, followed by B. coagulans $C 45$ and $B$. licheniformis A3; the control compost without inoculation had the lowest microbial populations.

The numbers of mesophilic and thermo-tolerant actinomycetes, fungi, phosphate-solubilizing actinomycetes and phosphate-solubilizing fungi increased gradually during 56 days composting. Compost inoculated with $S$. thermophilus J57 had the largest populations of mesophilic actinomycetes $\left(2.3 \pm 0.1 \times 10^{6} \mathrm{CFU} \mathrm{g}^{-1}\right)$, thermo-tolerant actinomycetes $\left(1.3 \pm 0.1 \times 10^{6} \mathrm{CFU} \mathrm{g^{-1 }}\right)$, mesophilic phosphate-solubilizing actinomycetes $\left(1.8 \pm 0.1 \times 10^{6} \mathrm{CFU} \mathrm{g}{ }^{-1}\right)$, and thermo-tolerant phosphate-solubilizing actinomycetes $\left(1.0 \pm 0.0 \times 10^{6} \mathrm{CFU} \mathrm{g}^{-1}\right)$, followed by $S$. thermonitrificans NTU-88, B. smithii F18, B. coagulans C45; the non-inoculated control compost had the lowest populations of mesophilic actinomycetes

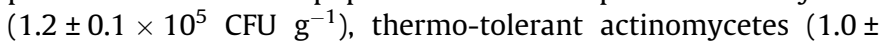
$\left.0.1 \times 10^{5} \mathrm{CFU} \mathrm{g}^{-1}\right)$, mesophilic phosphate-solubilizing actinomycetes $\left(7.8 \pm 1.0 \times 10^{5} \mathrm{CFU} \mathrm{g}^{-1}\right)$, and thermo-tolerant phosphatesolubilizing actinomycetes $\left(6.7 \pm 0.7 \times 10^{4} \mathrm{CFU} \mathrm{g}^{-1}\right)$.

Composts inoculated with $A$. fumigatus $\mathrm{O} 4$ had the significantly highest numbers of mesophilic fungi of $1.7 \pm 0.1 \times 10^{6} \mathrm{CFU} \mathrm{g}^{-1}$, thermo-tolerant fungi of $7.4 \pm 0.7 \times 10^{5} \mathrm{CFU} \mathrm{g}^{-1}$, mesophilic phosphate-solubilizing fungi of $1.4 \pm 0.1 \times 10^{6} \mathrm{CFU} \mathrm{g}^{-1}$, and thermo-tolerant phosphate-solubilizing fungi of $6.5 \pm 0.7 \times 10^{5} \mathrm{CFU} \mathrm{g}^{-1}$, followed by A. fumigatus NTU-132, B. smithii F18, and B. coagulans C45; the control compost without inoculation manifested the lowest numbers of mesophilic fungi of $8.9 \pm 0.4 \times 10^{5} \mathrm{CFU} \mathrm{g^{-1 }}$, thermo-tolerant fungi of $7.2 \pm 1.1 \times 10^{4} \mathrm{CFU} \mathrm{g}^{-1}$, mesophilic phosphate-solubilizing fungi of $7.5 \pm 0.2 \times 10^{5} \mathrm{CFU} \mathrm{g}^{-1}$, and thermo-tolerant phosphate-solubilizing fungi of $6.0 \pm 0.6 \times 10^{4}$ $\mathrm{CFU} \mathrm{g}^{-1}$.

Inoculations with $B$. coagulans $C 45, B$. licheniformis $A 3$, and $B$. smithii F18 increased the percentages of mesophilic phosphate-solubilizing bacteria and proteolytic microbes in the total mesophilic microbes, and thermo-tolerant phosphate-solubilizing bacteria in the total thermo-tolerant microbes from day 0 to day 7; these percentages dropped on day 14 , remained steady until day 28 , and then increased gradually until day 56 . After 56 days, compost inoculated with $B$. smithii $\mathrm{F} 18$ had the highest percentages of these microbes, followed by B. coagulans C45 and B. licheniformis A3 (Fig. 4).

Inoculation with $B$. smithii $\mathrm{F} 18$ also increased the percentages of thermo-tolerant proteolytic microbes in the total thermo-tolerant microbes between day 0 and day 7 , dropped on day 14 , remained steady until day 28 , and then gradually increased until day 56 . After 56 days, compost inoculated with B. smithii F18 had the highest percentages of these organisms, followed by B. coagulans $C 45$ and B. licheniformis A3 (Fig. 4).

The percentages of mesophilic actinomycetes, fungi, phosphatesolubilizing actinomycetes, and phosphate-solubilizing fungi in the total mesophilic microbes, and the percentages of thermo-tolerant actinomycetes, fungi, phosphate-solubilizing actinomycetes, and phosphate-solubilizing fungi in the total thermo-tolerant microbes during the first 14 days of composting dropped significantly to the lowest of $0.004-0.34 \%$. The populations of mesophilic and thermotolerant actinomycetes and fungi, mesophilic and thermo-tolerant phosphate-solubilizing actinomycetes and fungi had the highest on day 56 composting. The composts inoculated with $S$. thermophilus J57 manifested the highest percentages of mesophilic actinomycetes $(8.2 \pm 0.8 \%)$, thermo-tolerant actinomycetes (17.7 $\pm 1.9 \%)$,

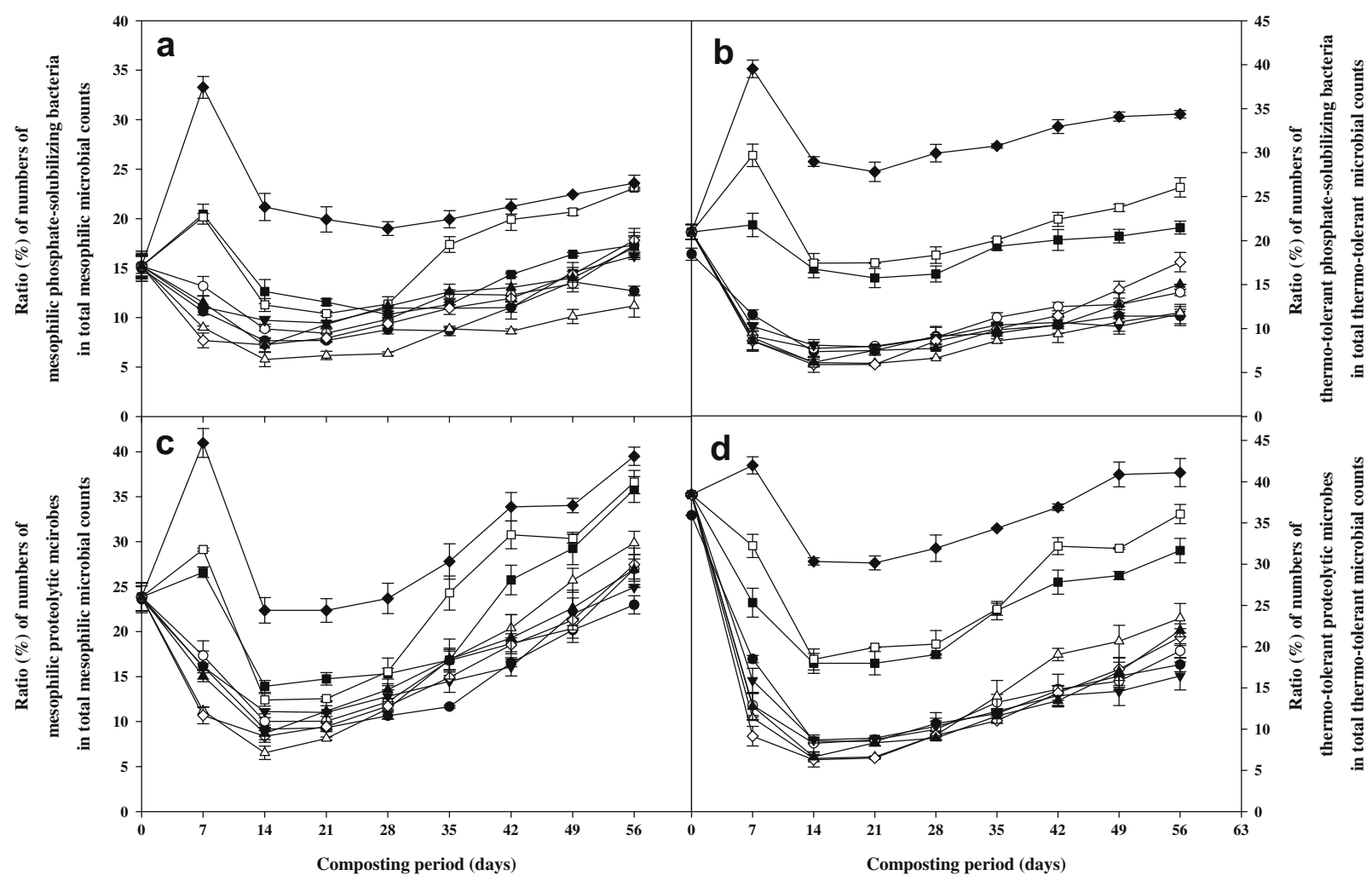

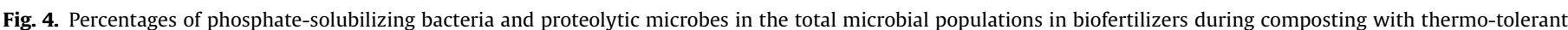

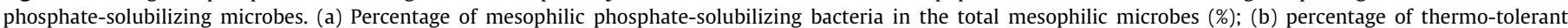

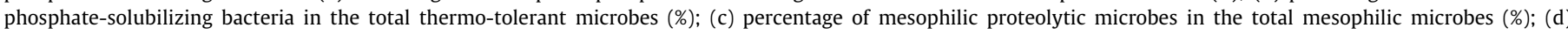

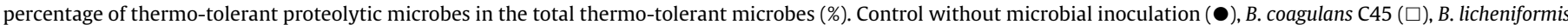

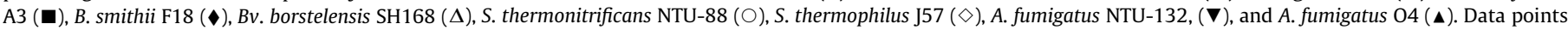
are means and vertical bars are standard deviations $(n \geqslant 3)$. 
mesophilic phosphate-solubilizing actinomycetes ( $6.3 \pm 0.7 \%)$, and thermo-tolerant phosphate-solubilizing actinomycetes (14.2 \pm $0.9 \%$ ), followed by $S$. thermonitrificans NTU-88, and the noninoculated control compost possessed the lowest percentages of mesophilic actinomycetes $(5.0 \pm 0.4 \%)$, thermo-tolerant actinomycetes $(3.3 \pm 0.3 \%)$, mesophilic phosphate-solubilizing actinomycetes $(3.4 \pm 0.1 \%)$, and thermo-tolerant phosphate-solubilizing actinomycetes $(2.2 \pm 0.3 \%)$.

The composts inoculated with A. fumigatus 04 presented the highest percentages of mesophilic fungi ( $6.4 \pm 0.9 \%)$, thermo-tolerant fungi $(11.0 \pm 0.9 \%)$, mesophilic phosphate-solubilizing fungi $(5.2 \pm 0.6 \%)$, and thermo-tolerant phosphate-solubilizing fungi (9.7 $\pm 1.0 \%)$; followed by A. fumigatus NTU-132; and the control compost without inoculation had the lowest percentages of mesophilic fungi $(3.9 \pm 0.4 \%)$, thermo-tolerant fungi $(2.3 \pm 0.4 \%)$, mesophilic phosphate-solubilizing fungi $(3.3 \pm 0.4 \%)$, and thermotolerant phosphate-solubilizing fungi $(1.9 \pm 0.2 \%)$.

The compost inoculated with thermo-tolerant phosphatesolubilizing bacterium $B$. smithii $\mathrm{F} 18$ had the highest soluble phosphorus content that helped microbial growth and composting organic wastes. Therefore, the composts manifested the highest numbers and proportions of mesophilic and thermo-tolerant phosphate-solubilizing and proteolytic microbes, and the fastest maturity rate. Same results were also found in thermo-tolerant phosphate-solubilizing actinomycete $S$. thermophilus J57 and thermo-tolerant phosphate-solubilizing fungus A. fumigatus $\mathrm{O}$.

\section{Conclusions}

Thermo-tolerant multi-functional phosphate-solubilizing microbes with six types of enzyme activities and three types of inorganic phosphate-solubilizing activities at 25 and $50{ }^{\circ} \mathrm{C}$ were isolated from the composts and biofertilizers. Inoculation with these microbes into compost accelerated the decomposition of agricultural and animal wastes during the preparation of biofertilizers with high soluble phosphorus content. Inoculation with $B$. smithii F18 had the shortest maturity period, the highest soluble phosphorus content, and the highest microbial populations, followed by B. coagulans $\mathrm{C} 45$ and B. licheniformis A3. Inoculation with thermo-tolerant phosphate-solubilizing bacteria released more soluble phosphorus than did inoculation with actinomycetes or fungi. Compost inoculated with thermo-tolerant phosphate-solubilizing bacteria might be a good strategy for biofertilizer preparation, and B. smithii F18 appears to be the best candidate for converting agricultural and animal wastes into biofertilizer. Mixed cultures of $B$. smithii F18 and other functional microbes can convert agricultural, animal and food wastes, vegetable and fruit market wastes, and poultry and livestock wastes into multi-functional biofertilizers for bioresource recycling and sustainable agriculture applications.

\section{Acknowledgements}

The authors thank Dr. I-Chu Chen, Mr. Yeu-Chuan Cheng and Miss Chia-Bei Wei for their technical assistances and the Council of Agriculture, Taiwan [92 Agri-4.2.2-Food-Z1(4), 93 Agri-4.1.6Food-22(4), 94 Agri-5.1.5-Food-Z1(3), 95 Agri-6.1.4-Food-Z1(2)] and National Science Council, Taiwan (NSC 92-2317-B005-021) for their financial supports.

\section{References}

Aquilanti, L., Favilli, F., Clementi, F., 2004. Comparison of different strategies for isolation and preliminary identification of Azotobacter from soil samples. Soil Biol. Biochem. 36, 1475-1483.

Arun, A.B., Sridhar, K.R., 2005. Growth tolerance of rhizobia isolated from sand dune legumes of the southwest coast of India. Eng. Life Sci. 5, 134-138.
Barnett, G.M., 1994. Phosphorus forms in animal manure. Bioresour. Technol. 49, 139-147.

Biswas, D.R., Narayanasamy, G., 2006. Rock phosphate enriched compost: an approach to improve low-grade Indian rock phosphate. Bioresour. Technol. 97, 2243-2251.

Chang, C.H., Hsieh, C.Y., Yang, S.S., 2001. Effect of cultural media on the phosphatesolubilizing activity of thermo-tolerant microbes. J. Biomass Energy Soc. China 20, 79-90.

Chang, C.H., Yang, S.S., 2006. Addition of polyelectrolyte oxygen detoxifier in poultry and livestock waste compost accelerating its maturity and maintaining population of thermo-tolerant microbes during composting. In: Proc. 6th Cross Strait Conf. Soil Sci. Fertil. vol. 2, pp. 677-692.

Council of Agriculture, 2007. Annual Report of Taiwan Agriculture-2006. Council of Agriculture, Executive Yuan, Taipei, Taiwan.

Del Campillo, M.C., Van der Zee, S.E.A.T.M., Torrent, J., 1999. Modelling long-term phosphorus leaching and changes in phosphorus fertility in excessively fertilized acid sandy soils. Eur. J. Soil Sci. 50, 391-399.

Delvasto, P., Valverde, A., Ballester, A., Igual, J.M., Muñoz, J.A., González, F., Blázquez, M.L., García, C., 2006. Characterization of brushite as a re-crystallization product formed during bacterial solubilization of hydroxyapatite in batch cultures. Soil Biol. Biochem. 38, 2645-2654.

Fageria, N.K., Baligar, V.C., 2001. Improving nutrient use efficiency of annual crops in Brazilian acid soils for sustainable crop production. Commun. Soil Sci. Plant Anal. 32, 1303-1319.

Haki, G.D., Rakshit, S.K., 2003. Developments in industrially important thermostable enzymes: a review. Bioresour. Technol. 89, 17-34.

Hegde, U., Chang, T.C., Yang, S.S., 2003. Methane and carbon dioxide emissions from Shan-Chu-Ku landfill site in northern Taiwan. Chemosphere 52, 1275-1285.

Huang, S.N., 1991. Application of hog compost in crop production. In: Proc. Semin. Hog Waste Treatment, Compost Preparation, Utilization and Management. The Biomass Energy Society of China, Taipei, Taiwan, pp. 1-17.

Johri, J.K., Surange, S., Nautiyal, C.S., 1999. Occurrence of salt, pH, and temperaturetolerant, phosphate-solubilizing bacteria in alkaline soils. Curr. Microbiol. 39, 89-93.

Kammoun, R., Naili, B., Bejar, S., 2008. Application of a statistical design to the optimization of parameters and culture medium for $\alpha$-amylase production by Aspergillus oryzae CBS 819.72 grown on gruel (wheat grinding by-product). Bioresour. Technol. 99, 5602-5609.

Lee, C.C., 1999. Organic waste treatment and sustainable agriculture. In: Huang, S.F., Lu, H.S., Chu, C. (Eds.), Treatment and Application of Agricultural Organic Wastes. The Biomass Energy Society of China, Taipei, Taiwan, pp. 1-7.

Lee, Y.J., Kim, B.K., Lee, B.H., Jo, K.I., Lee, N.K., Chung, C.H., Lee, Y.C., Lee, J.W., 2008. Purification and characterization of cellulase produced by Bacillus amyoliquefaciens DL-3 utilizing rice hull. Bioresour. Technol. 99, 378-386.

Liu, X.D., Xu, Y., 2008. A novel raw starch digesting $\alpha$-amylase from a newly isolated Bacillus sp. YX-1: purification and characterization. Bioresour. Technol. 99, 4315-4320.

Mahanta, N., Gupta, A., Khare, S.K., 2008. Production of protease and lipase by solvent tolerant Pseudomonas aeruginosa PseA in solid-state fermentation using Jatropha curcas seed cake as substrate. Bioresour. Technol. 99, 1729-1735.

Mandels, M., Medeiros, J.E., Andreotti, R.E., Bissett, F.H., 1981. Enzymatic hydrolysis of cellulose: evaluation of cellulase culture filtrates under use conditions. Biotechnol. Bioeng. 23, 2009-2026.

Mayende, L., Wilhelmi, B.S., Pletschke, B.I., 2006. Cellulase (CMCase) and polyphenol oxidase from thermophilic Bacillus spp. isolated from compost. Soil Biol. Biochem. 38, 2963-2966.

Nautiyal, C.S., 1999. An efficient microbiological growth medium for screening phosphate solubilizing microorganisms. FEMS Microbiol. Lett. 170, 265-270.

Nautiyal, C.S., Bhadauria, S., Kumar, P., Lal, H., Mondal, R., Verma, D., 2000. Stress induced phosphate solubilization in bacteria isolated from alkaline soils. FEMS Microbiol. Lett. 182, 291-296.

Olsen, S.R., Sommers, L.E., 1982. Phosphorus. In: Page, A.L., Miller, R.H., Keeney, D.R. (Eds.), Methods of Soil Analysis, Part 2, Chemical and Microbial Properties, second ed. American Society of Agronomy, Madison, Wisconsin, pp. 403-430.

Ouahmane, L., Thioulouse, J., Hafidi, M., Prin, Y., Ducousso, M., Galiana, A Plenchette, C., Kisa, M., Duponnois, R., 2007. Soil functional diversity and P solubilization from rock phosphate after inoculation with native or allochtonous arbuscular mycorrhizal fungi. For. Ecol. Manage. 241, 200-208.

Pai, C.R., Wu, C.F., Sun, R.Y., Wei, C.B., Yang, S.S., 2003. Composition analysis of livestock and poultry waste during composting. J. Biomass Energy Soc. China 22, 57-71.

Reddy, M.S., Kumar, S., Babita, K., Reddy, M.S., 2002. Biosolubilization of poorly soluble rock phosphates by Aspergillus tubingensis and Aspergillus niger. Bioresour. Technol. 84, 187-189.

Rodríguez, H., Fraga, R. 1999. Phosphate solubilizing bacteria and their role in plant growth promotion. Biotechnol. Adv. 17, 319-339.

Rudresh, D.L., Shivaprakash, M.K., Prasad, R.D., 2005. Tricalcium phosphate solubilizing abilities of Trichoderma spp. in relation to $\mathrm{P}$ uptake and growth and yield parameters of chickpea (Cicer arietinum L.). Can. J. Microbiol. 51, 217222.

SAS Institute, 2002. SAS/STAT User's Guide, Release 6.03. SAS Institute, Cary, North Carolina.

Saxena, R.K., Dutt, K., Agarwal, L., Nayyar, P., 2007. A highly thermostable and alkaline amylase from a Bacillus sp. PN5. Bioresour. Technol. 98, 260-265.

Shenoy, V.V., Kalagudi, G.M., 2005. Enhancing plant phosphorus use efficiency for sustainable cropping. Biotechnol. Adv. 23, 501-513. 
Takahashi, S., Anwar, M.R., 2007. Wheat grain yield, phosphorus uptake and soil phosphorus fraction after 23 years of annual fertilizer application to an Andosol. Field Crops Res. 101, 160-171.

Tanaka, Y., Murata, A., Hayashida, S., 1995. Accelerated composting of cereal shochu-distillery wastes by actinomycetes: promotive composting of shochudistillery wastes (I). Seibutsu-Kogaku Kaishi 73, 365-372.

Tsai, S.H., Liu, C.P., Yang, S.S., 2007. Microbial conversion of food wastes for biofertilizer production with thermophilic lipolytic microbes. Renew. Energy 32, 904-915.

Wakelin, S.A., Warren, R.A., Harvey, P.R., Ryder, M.H., 2004. Phosphate solubilization by Penicillium spp. Closely associated with wheat roots. Biol. Fertil. Soils 40, 36-43.

Yang, S.S., 2003. Application of microbial fertilizers on the three objectives agriculture. In: Chou, C.H., Yang, S.S. (Eds.), Challenge of Three Objectives in Agriculture. Council of Agriculture, Southern Taiwan Joint Services Center of Executive Yuan, Institute of Biotechnology of National Pingtung University of Science and Technology, Department of Biochemical Science and Technology of National Taiwan University, Taiwan, pp. 265-292.
Yang, S.S., Chang, H.L., Wei, C.B., Lin, H.C., 1991. Reduce waste production in Kjeldahl methods. J. Biomass Energy Soc. China 10, 147-155.

Yang, S.S., Chen, K.S., 2003. Application of thermophilic microbes for preparing biofertilizers. Plant Prot. Bull. Spe. Publ. New 5, 267-291.

Yang, S.S., Lin, C.F., Wang, C.K., 2003. Wastes Treatment and Re-Utilization, second ed. National Open University, Taipei, Taiwan, p. 501.

Zayed, G., Abdel-Motaal, H., 2005a. Bio-active composts from rice straw enriched with rock phosphate and their effect on the phosphorous nutrition and microbial community in rhizosphere of cowpea. Bioresour. Technol. 96, 929935.

Zayed, G., Abdel-Motaal, H., 2005b. Bio-production of compost with low pH and high soluble phosphorus from sugar cane bagasse enriched with rock phosphate. World J. Microbiol. Biotechnol. 21, 747-752.

Zou, X., Binkley, D., Doxtader, K.G., 1992. A new method for estimating gross phosphorus mineralization and immobilization rates in soils. Plant Soil 147, 243-250. 